\title{
HALL EFFECTS ON ISOTHERMAL VERTICAL PLATE WITH UNIFORM MASS DIFFUSION IN THE PRESENCE OF ROTATING FLUID AND CHEMICAL REACTION OF FIRST ORDER
}

\author{
V.S.A. DHANANJEYA KUMAAR \\ Department of Chemical Engineering \\ Sri Venkateswara College of Engineering \\ Pennalur, Sriperumbudur Taluk 602117, INDIA \\ E-mail: dhananjeyakumaar55@gmail.com \\ R. MUTHUCUMARASWAMY* \\ Department of Applied Mathematics \\ Sri Venkateswara College of Engineering \\ Pennalur, Sriperumbudur Taluk 602117, INDIA \\ E-mail: msamy@svce.ac.in
}

\begin{abstract}
An exact solution of the combined study of Hall effects on a vertical plate with a rotating fluid in the presence of a homogeneous chemical reaction of first order has been analysed. The dimensionless governing coupled partial differential equations are tackled using the usual Laplace transform technique. The sway of the Hall parameter, Hartmann number, Grashof number, Prandtl number, Schmidt number, chemical reaction parameter on the axial velocity and concentration of the fluid has been depicted graphically. When the non-dimensional angular velocity, $\Omega=\frac{2 M^{2}}{1+m^{2}}$, the transverse velocity component vanishes, thereby the axial velocity of the fluid attains the maximum value. It is noted that with increase in the Hall parameter, thermal Grashof number and mass Grashof number, the axial velocity of the fluid increases significantly.
\end{abstract}

Key words: Hall effect, chemical reaction parameter, rotating fluid, angular velocity.

\section{Introduction}

A magnetohydrodynamic flow refers to the flow of an electrically conducting fluid, such as plasma or ionised gas in the presence of a magnetic field. The flow of electrically conducting fluids in the presence of a magnetic field induces a potential in the direction normal to both electric and magnetic fields. This phenomenon is known as Hall effects. Thus, we can say that Hall effects are resulting phenomena of magnetohydrodynamics. The effect of the magnetic field on the flow of the fluid can be derived using Reynolds's transport theorem and Maxwell's equations. Pop [1] studied the effect of Hall current on a hydromagnetic flow, which is short lived. Gupta [2] investigated the hydromagnetic flow past a porous flat plate taking Hall effects into consideration. Ram [3] studied effects of Hall and ion-slip currents on free convective heat generating flow in a rotating fluid. Ghosh et al. [4] evaluated Hall effects on an MHD flow in a rotating system with heat transfer characteristics. Muthucumarswamy and Jeyanthi [5] studied the Hall effects on an MHD flow past an infinite vertical plate in a rotating fluid of variable temperature.

The effect of Hall current on fluids has received a humongous attention from researchers lately. This is due to its wide applications, varying from industrial level to the natural phenomena occurring at cosmological and geophysical level. The rotating flow of an electrically conducting fluid in the presence of a

\footnotetext{
* To whom correspondence should be addressed
} 
magnetic field is encountered in cosmical and geophysical fluid dynamics. It is well known that a number of astronomical bodies possess fluid interiors and magnetic fields. A star, a celestial body composed of plasma, is spherical due to the high internal pressure balanced by gravitational force. A large number of stars observed in the sky are either rotating stars or binary stars. These stars are subjected to the heliosphere in our solar system. According to Lal et al. [6]. The gravitational, centrifugal and Coriolis forces act on a star. These forces acting on the plasma of the rotating star, under the magnetic field influence, result in Hall effects. Considering a radioactive decay reaction in the star, which follows first order reaction kinetics, would make the mathematical studies of our problem correlate well with the phenomena discussed above.

Hall effects on an unsteady hydromagnetic flow past an accelerated porous plate in a rotating system was studied by Das et al. [7] Chemical reactions can be codified as either heterogeneous or homogeneous processes. This depends on whether they occur at an interface or as a single phase volume reaction. In wellmixed systems, the reaction is heterogeneous, if it takes place at an interface and homogeneous, if it takes place in a solution. Chambre and Young [8] analysed a first order chemical reaction in the neighbourhood of a horizontal plate.

In this paper, we study and investigate the combined outcome of Hall effect on an isothermal vertical plate with a rotating fluid following first order reaction kinetics. We study the effect of the Hall parameter $\mathrm{m}$, Hartmann number $\mathrm{M}$, rotation parameter $\Omega$, thermal Grashof number Gr, mass Grashof number Gc, Prandtl number Pr, Schmidt number Sc, chemical reaction parameter $K$ on the concentration and axial velocity of the fluid.

\section{Mathematical formulation of the problem}

An unsteady hydromagnetic flow of fluid past an infinite isothermal vertical plate with uniform mass diffusion has been presented. The fluid and the plate rotate in unison with a uniform angular velocity $\Omega^{\prime}$ about the $z^{\prime}$ - axis normal to the plate. Initially, the fluid is assumed to be at rest and surrounds an infinite vertical plate with temperature $T_{\infty}^{\prime}$ and concentration $C_{\infty}^{\prime}$. A magnetic field of uniform strength $B_{0}$ is transversely applied to the plate. The $x^{\prime}$-axis is taken along the plate in the vertically upward direction and the $z^{\prime}$ - axis is taken normal to the plate. At time $\frac{\partial C^{\prime}}{\partial t^{\prime}}=D \frac{\partial^{2} C^{\prime}}{\partial y^{2}}$, the plate and the fluid are at the same temperature $T_{\infty}^{\prime}$ in the stationary condition with the concentration level $C_{\infty}^{\prime}$ at all the points. At time $t^{\prime}>0$, the plate is exponentially accelerated with a velocity $u=u_{0}$ where the constant $u_{0}$ is the amplitude of the motion, $a^{\prime}$ is the accelerating parameter in its own plane and the plate temperature and concentration are raised to and $C_{w}^{\prime}$ and are maintained constantly thereafter. All the physical properties of the fluid are considered to be constant except the influence of the body force term. Then under the usual Boussinesq's approximation the unsteady flow equations are the momentum equation, energy equation, and mass equation, respectively.

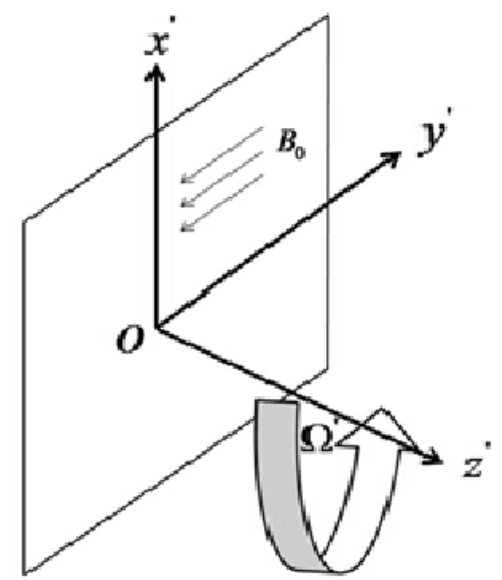

Fig.1. Mathematical model of the problem. 
Equation of momentum

$$
\begin{aligned}
& \frac{\partial u}{\partial t^{\prime}}-2 \Omega^{\prime} v=v \frac{\partial^{2} u}{\partial z^{2}}-\frac{1}{\rho} \frac{\partial p}{\partial x}+g+\frac{B_{0}}{\rho} j_{y} \\
& \frac{\partial v}{\partial t^{\prime}}+2 \Omega^{\prime} u=v \frac{\partial^{2} v}{\partial z^{2}}-\frac{B_{0}}{\rho} j_{x}
\end{aligned}
$$

Equation of energy

$$
\rho C_{p} \frac{\partial T^{\prime}}{\partial t^{\prime}}=k \frac{\partial^{2} T^{\prime}}{\partial z^{2}}
$$

Equation of diffusion

$$
\frac{\partial C^{\prime}}{\partial t^{\prime}}=D \frac{\partial^{2} C^{\prime}}{\partial z^{2}}
$$

Since there is no large velocity gradient here, the viscous term in Eq.(2.1) vanishes for small and hence for the outer flow, besides there is no magnetic field along the $x$-direction gradient, so we have

$$
0=-\frac{\partial p}{\partial x}-\rho_{\infty} g
$$

By eliminating the pressure term from Eqs (2.1) and (2.5), we obtain

$$
\frac{\partial u}{\partial t^{\prime}}-2 \Omega^{\prime} v=v \frac{\partial^{2} u}{\partial z^{2}}+\left(\rho_{\infty}-\rho\right) g+\frac{B_{0}}{\rho} j_{y}
$$

The Boussinesq approximation gives

$$
\rho_{\infty}-\rho=\rho_{\infty} \beta\left(T^{\prime}-T_{\infty}^{\prime}\right)+\rho_{\infty} \beta^{*}\left(C^{\prime}-C_{\infty}^{\prime}\right)
$$

On using Eq.(2.7) in Eq.(2.6) and noting that $\rho_{\infty}$ is approximately equal to 1, the momentum equation reduces to

$$
\frac{\partial u}{\partial t^{\prime}}-2 \Omega^{\prime} v=v \frac{\partial^{2} u}{\partial z^{2}}+\frac{B_{0}}{\rho} j_{y}+g \beta\left(T^{\prime}-T_{\infty}^{\prime}\right)+g \beta^{*}\left(C^{\prime}-C_{\infty}^{\prime}\right)
$$

The generalized Ohm's law, on taking Hall currents into account and neglecting ion-slip and thermoelectric effect, is

$$
\boldsymbol{j}+\frac{\omega_{e} \tau_{e}}{B_{0}}(\boldsymbol{j} \times \boldsymbol{B})=\sigma(\boldsymbol{E}+\boldsymbol{q} \times \boldsymbol{B}) .
$$


Equation (2.9) gives

$$
\begin{aligned}
& j_{x}-\mathrm{m} j_{y}=\sigma v B_{0}, \\
& j_{y}+\mathrm{m} j_{x}=-\sigma u B_{0}
\end{aligned}
$$

where $\mathrm{m}=\omega_{e} \tau_{e}$ is the Hall parameter. Solving Eqs (2.10) and (2.11) for $j_{x}$ and $j_{y}$, we have

$$
\begin{aligned}
& j_{x}=\frac{\sigma B_{0}}{1+m^{2}}(v-\mathrm{m} u), \\
& j_{y}=\frac{\sigma B_{0}}{1+m^{2}}(u+\mathrm{m} v) .
\end{aligned}
$$

On the use of Eqs (2.12) and (2.13), the momentum Eqs (2.8) and (2.2) become

$$
\begin{aligned}
& \frac{\partial u}{\partial t^{\prime}}=v \frac{\partial^{2} u}{\partial z^{2}}+2 \Omega_{z} v-\frac{\sigma \mu_{e}^{2} H_{0}^{2}}{\rho\left(1+m^{2}\right)}(u+\mathrm{m} v)+g \beta\left(T-T_{\infty}\right)+g \beta^{*}\left(C^{\prime}-C_{\infty}^{\prime}\right), \\
& \frac{\partial v}{\partial t^{\prime}}=v \frac{\partial^{2} v}{\partial z^{2}}-2 \Omega_{z} u-\frac{\sigma \mu_{e}^{2} H_{0}^{2}}{\rho\left(1+m^{2}\right)}(\mathrm{m} u-v), \\
& \rho C_{p} \frac{\partial T}{\partial t^{\prime}}=k \frac{\partial^{2} T}{\partial z^{2}}, \\
& \frac{\partial C^{\prime}}{\partial t^{\prime}}=D \frac{\partial^{2} C^{\prime}}{\partial z^{2}}-K_{t}\left(C^{\prime}-C_{\infty}^{\prime}\right) .
\end{aligned}
$$

Here the second term on the right side of Eqs (2.14) and (2.15) are due to small Coriolis force. The boundary conditions are given by

$$
\begin{aligned}
& u=0, \quad T=T_{\infty}, \quad C^{\prime}=C_{\infty}^{\prime}, \quad v=0 \text { for all } z, t^{\prime} \leq 0, \\
& u=u_{0}, \quad T=T_{w}, \quad C^{\prime}=C_{w}^{\prime}, \quad v=0 \text { at } z=0 \text { for all } t^{\prime}>0, \\
& u \rightarrow 0, \quad T \rightarrow T_{\infty}, C^{\prime} \rightarrow C_{\infty}^{\prime} \text { as } z \rightarrow \infty \text { for all } t^{\prime}>0 .
\end{aligned}
$$

The dimensionless quantities are introduced as follows.

$$
\begin{aligned}
& U=\frac{u}{u_{0}}, \quad V=\frac{v}{u_{0}}, \quad t=\frac{t^{\prime} u_{0}^{2}}{v}, \quad Z=z\left(\frac{u_{0}^{2}}{v^{2}}\right), \quad \Omega=\Omega_{z}\left(\frac{v}{u_{0}^{2}}\right), \quad M^{2}=\frac{\sigma \mu_{e}^{2} H_{0}^{2} v}{2 \rho u_{0}^{2}}, \\
& \mathrm{Gr}=\frac{v g \beta\left(T_{w}-T_{\infty}\right)}{u_{0}^{3}}, \quad \mathrm{Gc}=\frac{v g \beta^{*}\left(C^{\prime}{ }_{w}-C^{\prime}{ }_{\infty}\right)}{u_{0}^{3}}, \quad \operatorname{Pr}=\frac{\mu c_{p}}{k}, \quad K=K_{l}\left(\frac{v}{u_{0}^{2}}\right) .
\end{aligned}
$$


Together with Eqs (2.1), (2.2), (2.3) and (2.4), boundary conditions Eqs (2.18), (2.19) using Eqs (2.20), we have

$$
\begin{aligned}
& \frac{\partial U}{\partial t}=\frac{\partial^{2} U}{\partial Z^{2}}+2 V\left(\Omega-\frac{M^{2} \mathrm{~m}}{1+\mathrm{m}^{2}}\right)-\frac{2 M^{2}}{1+\mathrm{m}^{2}} U+\theta \mathrm{Gr}+C \mathrm{Gc}, \\
& \frac{\partial V}{\partial t}=\frac{\partial^{2} V}{\partial Z^{2}}-2 U\left(\Omega+\frac{M^{2} \mathrm{~m}}{1+\mathrm{m}^{2}}\right)+\frac{2 M^{2} V}{1+\mathrm{m}^{2}}, \\
& \frac{\partial \theta}{\partial t}=\frac{1}{\operatorname{Pr}} \frac{\partial^{2} \theta}{\partial Z^{2}}, \\
& \frac{\partial C}{\partial t}=\frac{1}{\mathrm{Sc}} \frac{\partial^{2} C}{\partial Z^{2}}-K C,
\end{aligned}
$$

with the boundary conditions

$$
\begin{aligned}
& U=0, \quad \theta=0, C=0, V=0 \text { for all } Z, \quad t \leq 0, \\
& U=1, \quad \theta=1, \quad C=1, \quad V=0 \text { at } Z=0 \text { for all } t>0, \\
& U \rightarrow 0, \quad \theta \rightarrow 0, \quad C \rightarrow 0, \quad V \rightarrow 0 \text { as } Z \rightarrow \infty \text { for all } t>0
\end{aligned}
$$

Now Eqs (2.21), (2.22) and the boundary conditions (2.23), (2.24) can be combined to give,

$$
\frac{\partial F}{\partial t}=\frac{\partial^{2} F}{\partial Z^{2}}-F a+\theta \mathrm{Gr}+C \mathrm{Gc}
$$

where $\quad F=U+i V \quad$ and $\quad a=2\left[\frac{M^{2} \mathrm{~m}}{1+\mathrm{m}^{2}}+i\left(\Omega-\frac{M^{2} \mathrm{~m}}{1+\mathrm{m}^{2}}\right)\right]$.

In our study the value of $\Omega$ (rotation parameter) is taken as $\Omega=\frac{M^{2} m}{1+m^{2}}$, as a result of this the transverse velocity vanishes.

With the boundary conditions,

$$
\begin{aligned}
& F=0, \quad \theta=0, \quad C=0 \text { for all } Z, t \leq 0, \\
& F=1, \quad \theta=1, \quad C=1 \text { at } Z=0 \text { and for all } t>0, \\
& F \rightarrow 0, \quad \theta \rightarrow 0, \quad C \rightarrow 0 \text { as } Z \rightarrow \infty \text { for all } t>0 .
\end{aligned}
$$




\section{Method of solution}

The dimensionless governing Eqs (2.3), (2.4) and (2.25) subject to the initial and boundary conditions (2.26) are solved by the Laplace transform technique and the solutions are obtained as follows.

$$
\begin{aligned}
& \theta(Z, t)=\operatorname{erfc}(\eta \sqrt{\operatorname{Pr}}) \\
& C(Z, t)=\frac{1}{2}[\exp (2 \eta \sqrt{K t \mathrm{Sc}}) \operatorname{erfc}(\eta \sqrt{\mathrm{Sc}}+\sqrt{K t})+\exp (-2 \eta \sqrt{K t \mathrm{Sc}}) \operatorname{erfc}(\eta \sqrt{\mathrm{Sc}}-\sqrt{K t})], \\
& F(Z, t)=\frac{1}{2}[\exp (-2 \eta \sqrt{a t}) \operatorname{erfc}(\eta-\sqrt{a t})+\exp (2 \eta \sqrt{a t}) \operatorname{erfc}(\eta+\sqrt{a t})]+ \\
& +\frac{G r}{a}\left\{\begin{array}{l}
\frac{-1}{2}[\exp (-2 \eta \sqrt{a t}) \operatorname{erfc}(\eta-\sqrt{a t})+\exp (2 \eta \sqrt{a t}) \operatorname{erfc}(\eta+\sqrt{a t})]+ \\
+\frac{\exp (\gamma t)}{2}[\exp (-2 \eta \sqrt{(\gamma+a) t}) \operatorname{erfc}(\eta-\sqrt{(\gamma+a) t})+ \\
+\exp (2 \eta \sqrt{(\gamma+a) t}) \operatorname{erfc}(\eta+\sqrt{(\gamma+a) t})]+\operatorname{erfc}(\eta \sqrt{\operatorname{Pr}})+ \\
-\frac{\exp (\gamma t)}{2}[\exp (-2 \eta \sqrt{\operatorname{Pr} \gamma t}) \operatorname{erfc}(\eta \sqrt{\operatorname{Pr}}-\sqrt{\gamma t})+\exp (2 \eta \sqrt{\gamma \operatorname{Pr} t}) \operatorname{erfc}(\eta \sqrt{\operatorname{Pr}}+\sqrt{\gamma t})]
\end{array}\right\}+ \\
& \left(\frac{-1}{2}[\exp (-2 \eta \sqrt{a t}) \operatorname{erfc}(\eta-\sqrt{a t})+\exp (2 \eta \sqrt{a t}) \operatorname{erfc}(\eta+\sqrt{a t})]+\right. \\
& +\frac{1}{2}[\exp (-2 \eta \sqrt{\mathrm{ScKt}}) \operatorname{erfc}(\eta \sqrt{\mathrm{Sc}}-\sqrt{K t})+\exp (2 \eta \sqrt{\operatorname{Sc} K t}) \operatorname{erfc}(\eta \sqrt{\mathrm{Sc}}+\sqrt{K t})]+ \\
& +\frac{\mathrm{Gc}}{(a-\mathrm{Sc} K)}\left\{\begin{array}{l}
+\frac{\exp (\alpha t)}{2}[\exp (-2 \eta \sqrt{(a+\alpha) t}) \operatorname{erfc}(\eta-\sqrt{(a+\alpha) t})+ \\
+\exp (2 \eta \sqrt{(a+\alpha) t}) \operatorname{erfc}(\eta+\sqrt{(a+\alpha) t})]+
\end{array}\right. \\
& \left\{\begin{array}{l}
-\frac{\exp (\alpha t)}{2}[\exp (-2 \eta \sqrt{\operatorname{Sc}(K+\alpha) t}) \operatorname{erfc}(\eta \sqrt{\operatorname{Sc}}-\sqrt{(K+\alpha) t})+ \\
+\exp (2 \eta \sqrt{\operatorname{Sc}(K+\alpha) t}) \operatorname{erfc}(\eta \sqrt{\operatorname{Sc}}+\sqrt{(K+\alpha) t})]
\end{array}\right.
\end{aligned}
$$

where $\quad \gamma=\frac{a}{\operatorname{Pr}-1}, \quad \alpha=\frac{a-\mathrm{Sc} K}{\mathrm{Sc}-1}, \quad \eta=\frac{z}{2 \sqrt{t}}$.

In order to get a physical insight into the problem, the numerical values of $F(Z, t), C(Z, t), \theta(Z, t)$ have been computed and represented in a graphical form.

\section{Results and discussion}

For physical understanding of the problem, the effect of multiple parameters like $\mathrm{Gr}, \mathrm{Gc}, \mathrm{Sc}, t, K, m$, $M$ upon the flow and concentration profile are computed and presented graphically. For this purpose the time $t$ is taken as 2 and the value of the Prandtl number $\operatorname{Pr}$ as 0.7 and 7. The numerical values of the velocity, temperature and concentration fields are computed for different physical parameters like the Prandtl number, 
rotation parameter, Hartmann number, Hall parameter, thermal Grashof number, mass Grashof number, reaction parameter, Schmidt number and time

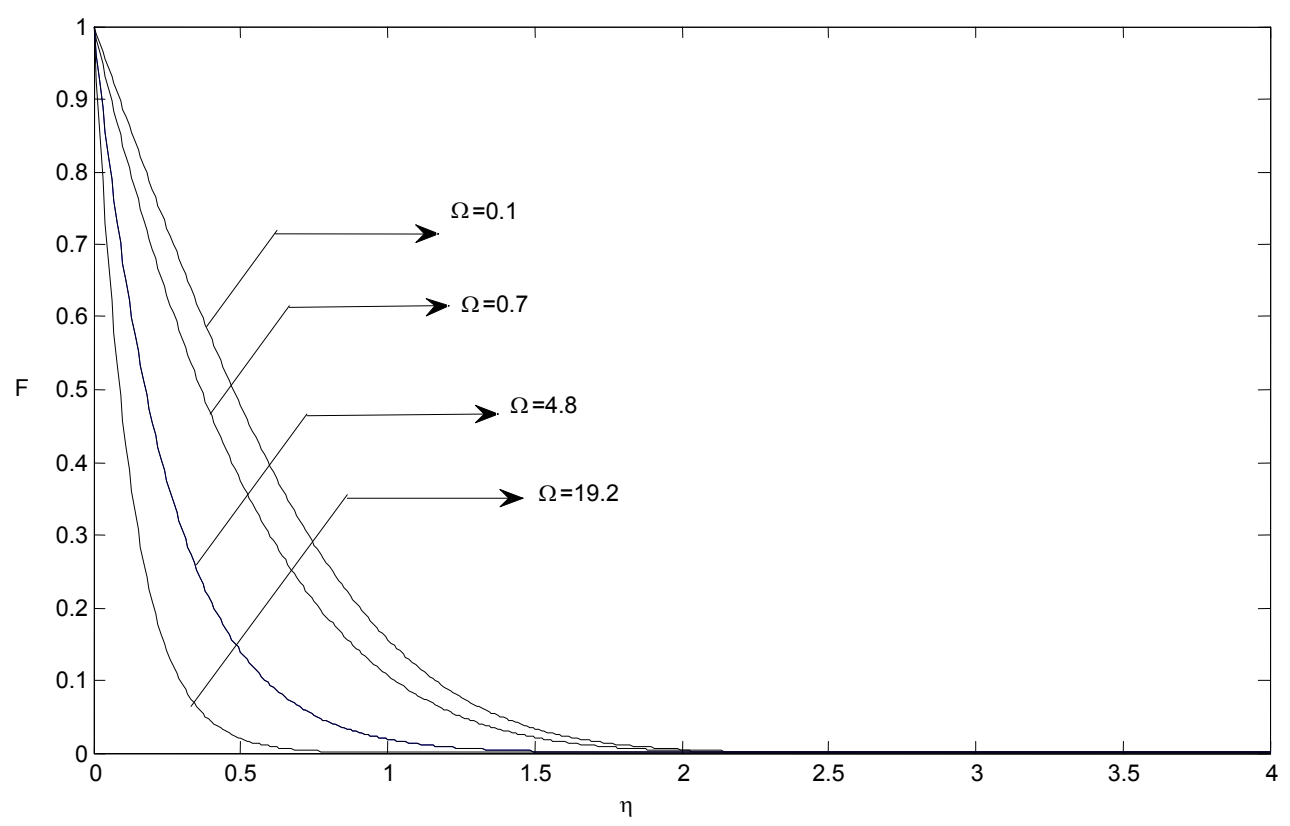

Fig.2. Axial velocity profile for different $\Omega$.

Figure 2 shows the axial velocity profile for various values of the rotation parameter $(\Omega=0.1,0.7$, 4.8, 19.2), $\mathrm{Sc}=2.01, \mathrm{~m}=5, \mathrm{Pr}=7, K=0.2, \mathrm{Gr}=5, \mathrm{Gc}=10$ and $t=2$ in the presence of water. It is observed that the axial velocity $F$ decreases with an increase in $\Omega$. Also, when $\Omega=\frac{M^{2} m}{1+m^{2}}$, the transverse velocity component vanishes and the axial velocity attains the maximum value (Barali and Borkakati [9])

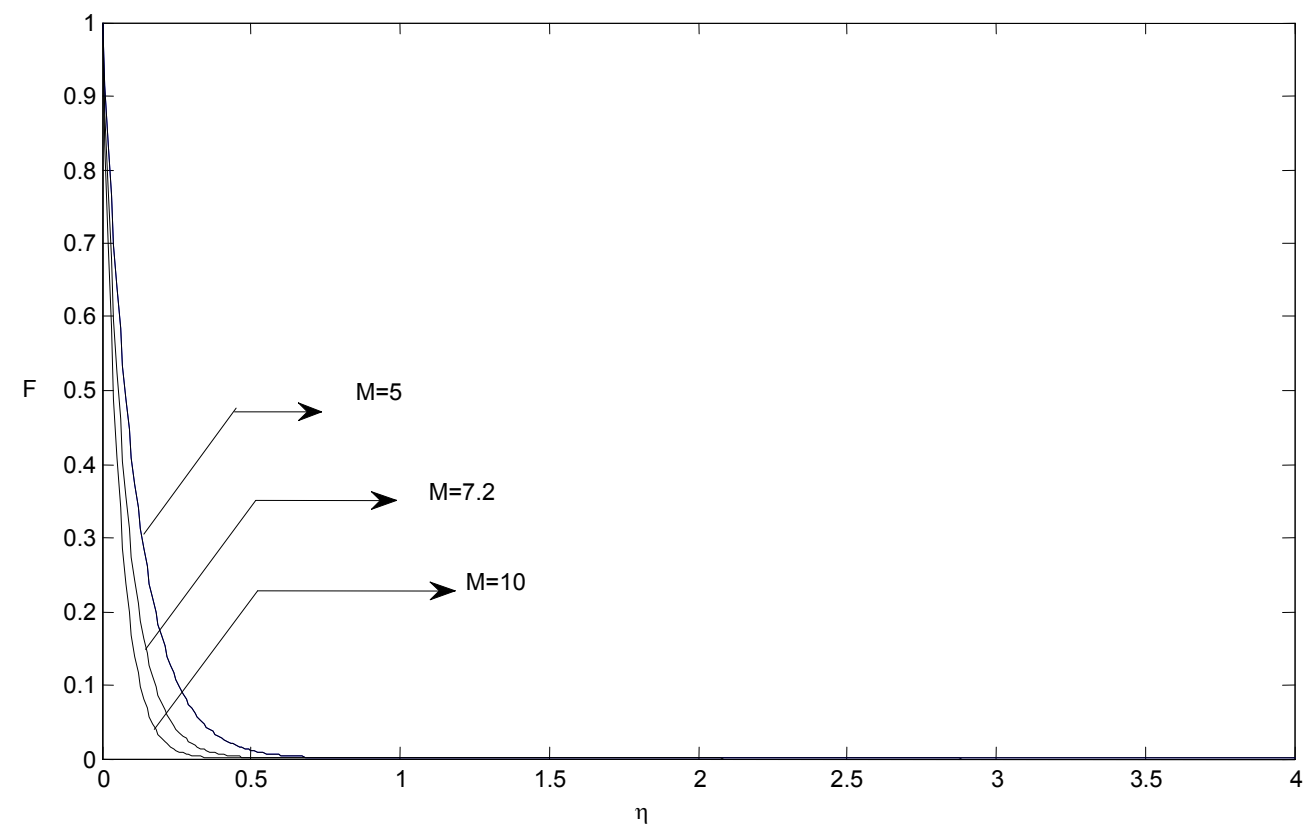

Fig.3. Axial velocity profile for different values of the Hartmann number, M. 
The effect of the Hartman number $(\mathrm{M}=5,7.2,10)$ on the axial velocity is shown in Fig.3 for the rotation parameter $\Omega=10, \mathrm{~m}=2, t=2, \mathrm{Sc}=2.01, \mathrm{Pr}=7, \mathrm{~K}=0.2, \mathrm{Gr}=5, \mathrm{Gc}=10$ in the presence of water. It is observed that with an increase in the Hartmann number (M) the axial velocity decreases.

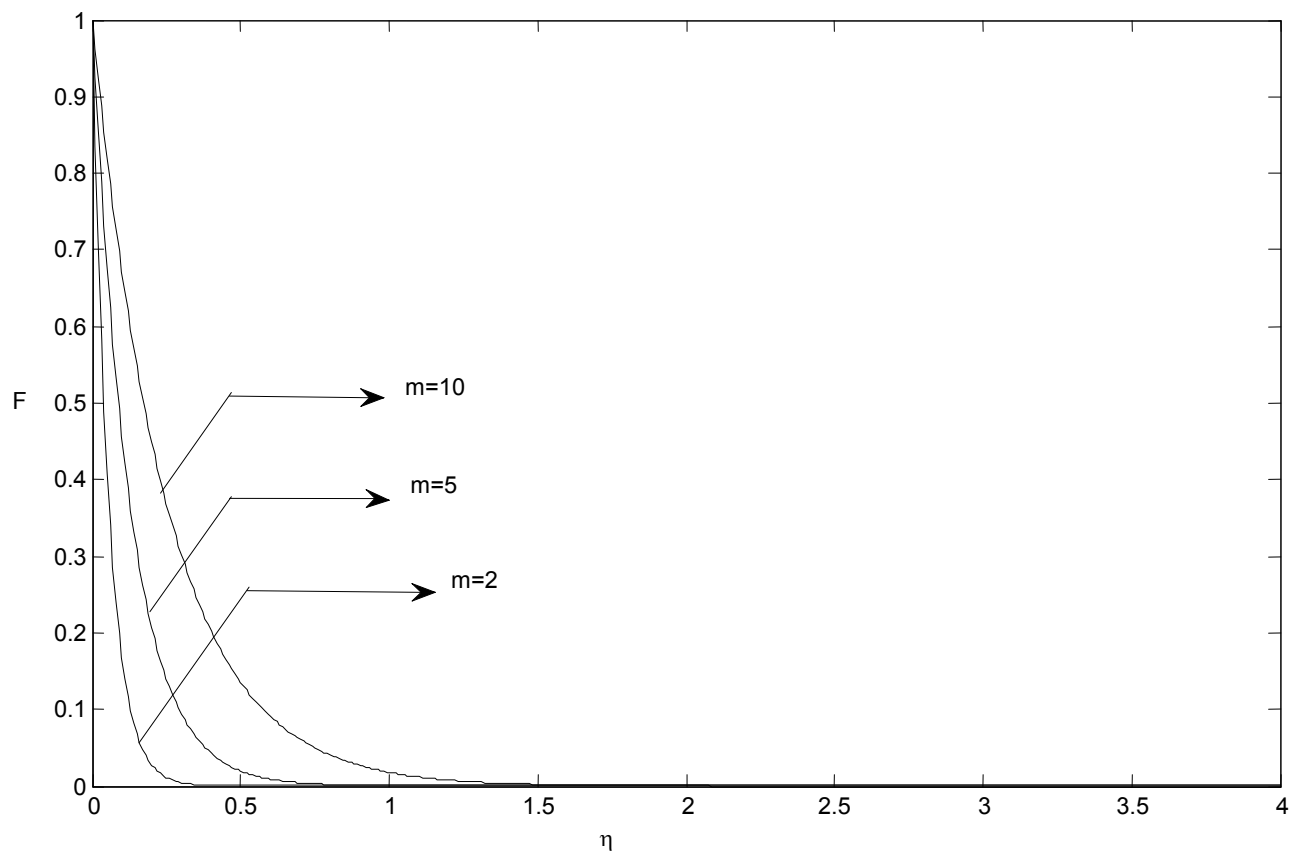

Fig.4. Axial velocity profile for different values of the Hall parameter, m.

Figure 4 shows the axial velocity profile for different values of the Hall parameter $(\mathrm{m}=10,5,2)$, with $\Omega=10, \mathrm{M}=10, t=2, \mathrm{Sc}=2.01, \mathrm{Pr}=7, K=0.2, \mathrm{Gr}=5, \mathrm{Gc}=10$ in the presence of water. It is noted that the axial velocity increases with increase in Hall parameter $\mathrm{m}$.

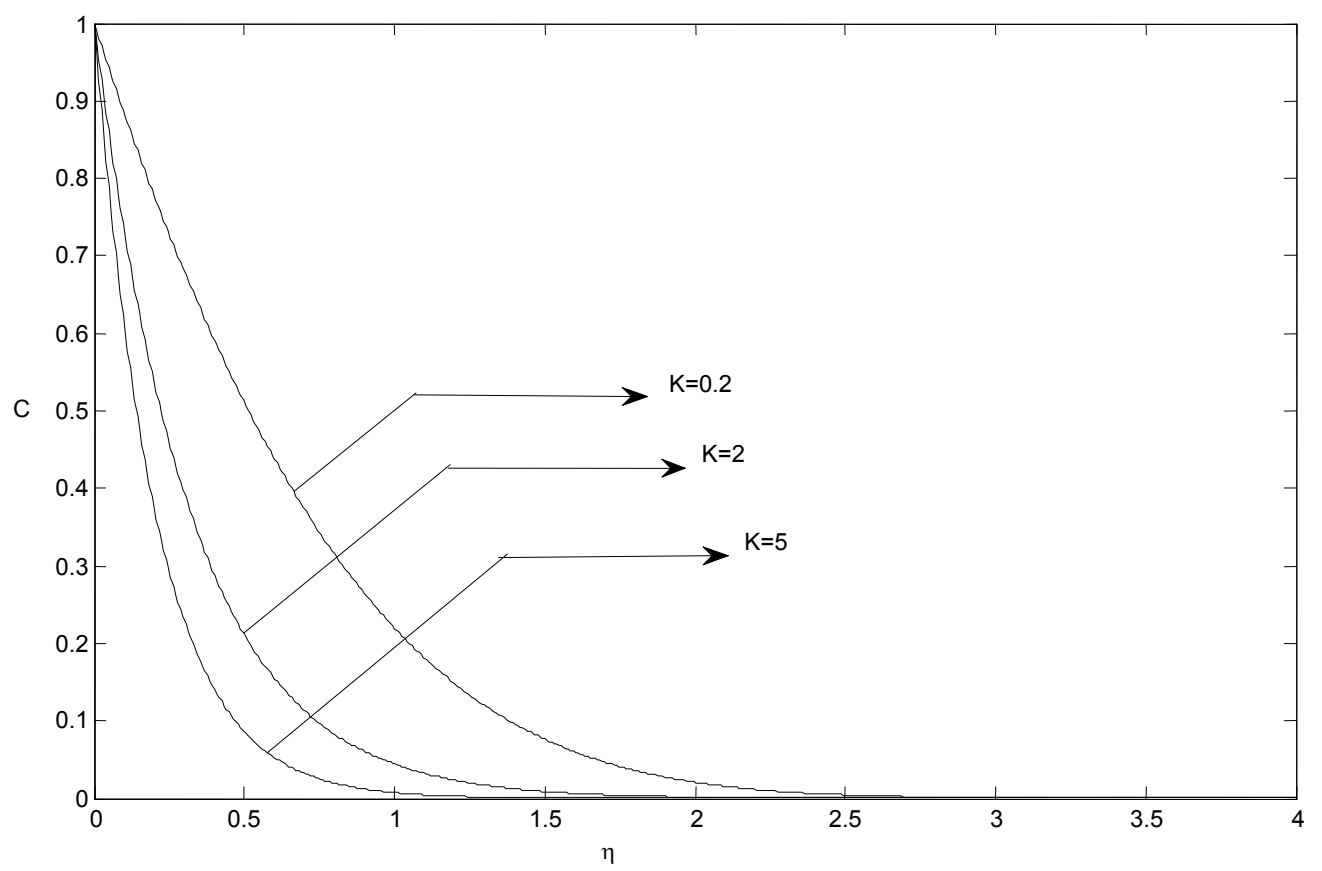

Fig.5. Concentration profile for different values of the reaction parameter, $K$. 
Figure 5 shows the effect of different values of the reaction parameter $(K=0.2,2,5)$ on the concentration profile with $\mathrm{Sc}=0.6$ and $t=2$. It is observed that the reaction parameter $K$ plays a crucial role in the concentration profile as shown above. With an increase in $K$, the concentration field decreases.

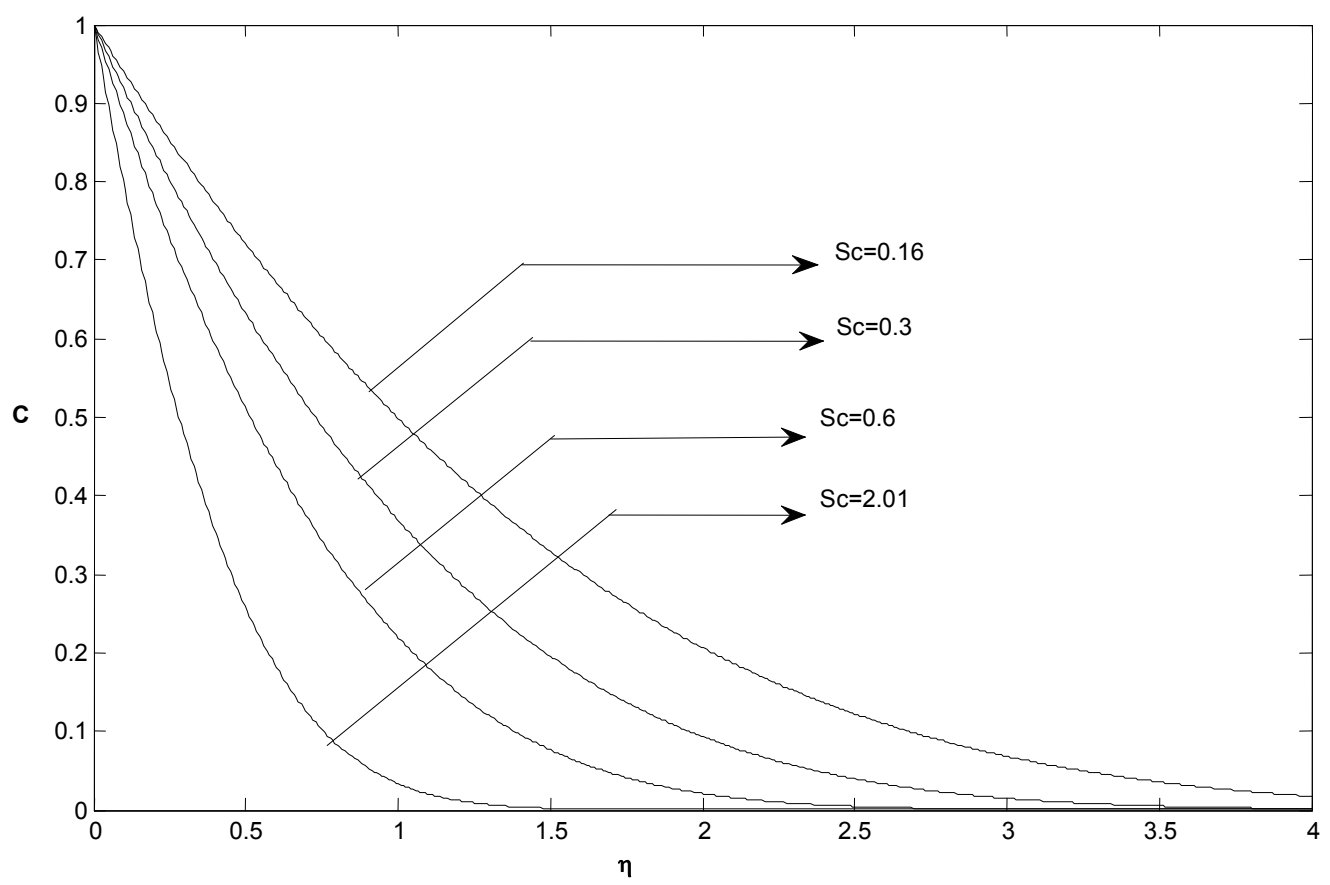

Fig.6. Concentration profile for different values of the Schmidt number, Sc.

Figure 6 shows the variation in the concentration profile with different values of the Schmidt number Sc with the reaction parameter $K=0.2$ and time $t=2$. It is observed that the concentration increases for decreasing values of the Schmidt number Sc.

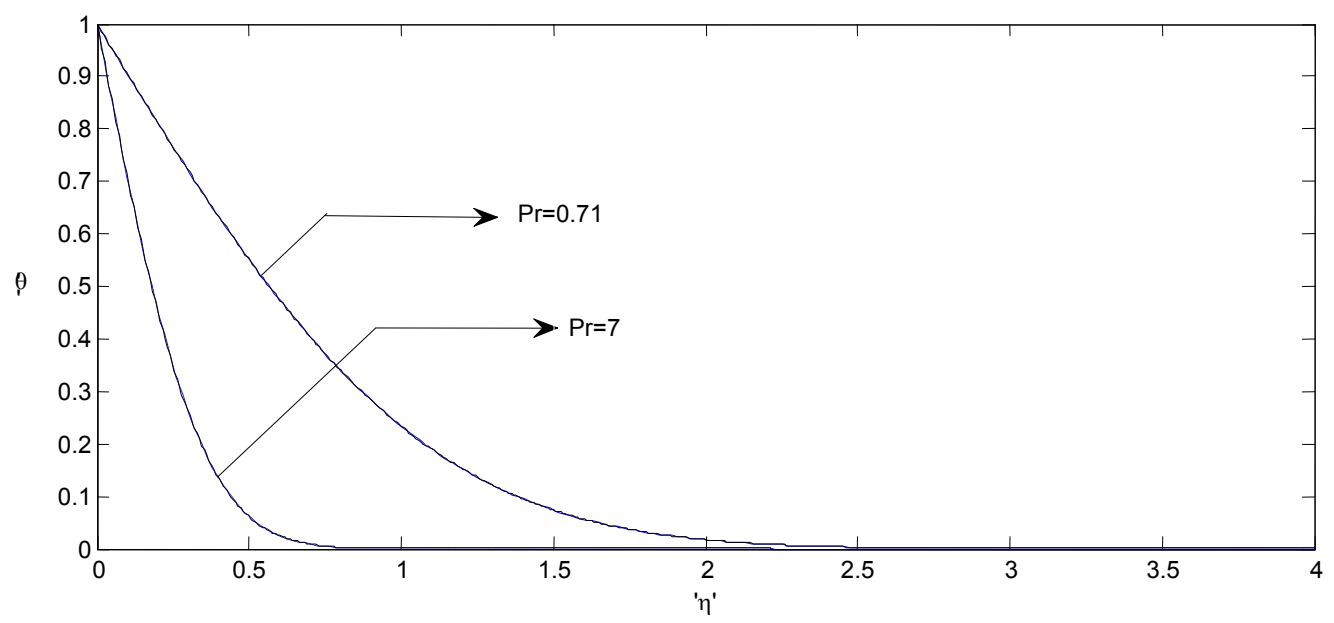

Fig.7. Temperature profile for different values of the Prandtl number, Pr.

The effect of $\operatorname{Pr}$ on the temperature profile is shown Fig.7. It is observed that the temperature increases with decreasing values of the Prandtl number. This shows that the heat transfer is more predominant in air when compared with water. 


\section{Conclusion}

A combined study of Hall effects on a vertical plate with a rotating fluid following first order reaction kinetics has been made. The effects of the thermal Grashof number, mass Grashof number, reaction parameter, rotation parameter, Hall parameter, Hartmann number on the concentration and axial velocity are presented graphically. When the rotation parameter $\Omega=\frac{M^{2} m}{1+m^{2}}$, the transverse velocity component vanishes so that the fluid moves axially. It is observed that with an increase in the Hall parameter, the axial velocity of the rotating fluid increases and the axial velocity decreases with an increase in the rotating parameter and Hartmann number. Such a study is found useful in cosmological applications.

\section{Nomenclature}

$B_{0} \quad$ - imposed magnetic field

$C$ - dimensionless concentration

$C^{\prime}$ - species concentration in the fluid

$C_{\infty}$ - concentration far away from the plate

$c_{w}$ - wall concentration

Gc - mass Grashof's number

$\mathrm{Gr}$ - thermal Grashof's number

$J_{z} \quad$ - component of current density $\mathrm{j}$

$K$ - chemical reaction parameter

$k$ - thermal conductivity

M - Hartman number

$\mathrm{m}$ - Hall parameter

Pr - thermal Prandtl number

Sc - Schmidt number

$T$ - temperature of the fluid near the plate

$T_{w} \quad$ - temperature of the plate

$T_{\infty} \quad$ - temperature of the fluid far away from the plate

$t$ - non-dimensional time

$t$, time

$(U, V, W)$ - non-dimensional velocity components

$(u, v, w)$ - components of velocity field $F$

$(x, y, z) \quad$ - Cartesian co-ordinates

$\beta-$ volumetric coefficient of thermal expansion

$\beta^{*} \quad-$ volumetric coefficient of expansion with concentration

$\theta$ - dimensionless temperature

$\mu \quad-$ coefficient of viscosity

$v-$ kinematic viscosity

$\rho-$ fluid density

$\sigma \quad$ - electrical conductivity

$\Omega$ - non-dimensional angular velocity

$\Omega_{z} \quad$ - component of angular viscosity

\section{References}

[1] Barali A. and Borkakati A.K. (1982): The effect of Hall current on MHD flow and heat transfer between two parallel porous plates. - Applied Scientific Research, vol.39, pp.155-165. 
[2] Chambre P.L. and Young J.D. (1958): On the diffusion of a chemically reactive species in a laminar boundary layer flow. - The Physics of Fluids, vol.1, pp.48-54.

[3] Das et al (2015): Hall effects on unsteady hydromagnetic flow past an accelerated porous plate in a rotating system. - Journal of Applied Fluid Mechanics, vol.8, No.3, pp.409-417. 9p.

[4] Deka R.K. (2008): Hall effects on MHD flow past an accelerated plate. - Theoretical Appl. Mech., vol.35, No.4, pp.333-346.

[5] Ghosh S.K. at al. (2009): Hall effects on MHD flow in a rotating system with heat transfer characteristics. Meccanica, vol.44, pp.741-765.

[6] Gupta S. (1975): Hydromagnetic flow past a porous flat plate with Hall effect. - Acta Mechanica, vol.22, pp.28128.

[7] Hetnarski R.B. (1975): An algorithm for generating some inverse Laplace transforms of exponential form. ZAMP 26, pp.249-253.

[8] Lal A.K. et al. (2009): Effect of Coriolis force on the shapes of rotating stars and stars in binary systems. Astrophys Space Sci., vol.319, pp.45-53.

[9] Muthucumarswamy R. and Jeyanthi (2014): Hall Effects on MHD flow past an infinite vertical plate in the presence of rotating fluid of variable temperature and uniform mass diffusion with first order chemical reaction. - International Journal of Applied Engineering Research, vol.9, pp.26259-26271.

[10] Pop I. (1971): The effect of Hall current on hydromagnetic flow near on accelerated plate. - J. Math. Phys. Sci., vol.5, pp.375-379.

[11] Ram P.C. (1995): Hall effect on hydromagnetic convection flow in a rotating fluid. - Astrophysics and Space Science, vol.158, No.2, pp.189-195.

Received: September 28, 2015

Revised: June 5, 2016 